\title{
Effects of osteoporosis on the biomechanics of various supplemental fixations co-applied with oblique lumbar interbody fusion (OLIF): a finite element analysis
}

\section{Zi-Xuan Liu}

Tianjin University of Technology

Zi-Wei Gao

Tianjin Hospital

Chao Chen

Tianjin Hospital

Zi-Yang Liu

Tianjin Hospital

Xin-Yi Cai

Tianjin University of Technology

Ya-Nan Ren

Tianjin University of Technology

\section{Xun Sun}

Tianjin Hospital

Xin-Long Ma

Tianjin Hospital

\section{Cheng-Fei Du}

Tianjin University of Technology

Qiang Yang ( $\triangle$ yangqiang1980@126.com )

Tianjin Hospital

\section{Research Article}

Keywords: Finite element analysis, Osteoporosis, Oblique lumbar interbody fusion, Various supplemental fixations, Biomechanical

Posted Date: February 25th, 2022

DOl: https://doi.org/10.21203/rs.3.rs-944717/v1 
License: (c) (i) This work is licensed under a Creative Commons Attribution 4.0 International License. Read Full License 


\section{Abstract}

Background: Oblique lumbar interbody fusion (OLIF) is an important surgical modality for the treatment of degenerative lumbar spine disease. Various supplemental fixations can be co-applied with OLIF, increasing OLIF stability and reducing complications. However, it is unclear whether osteoporosis affects the success of supplemental fixations; therefore, this study analyzed the effects of osteoporosis on various supplemental fixations co-applied with OLIF.

Methods: We developed and validated an L3-S1 finite element (FE) model; we assigned different material properties to each component and established models of the osteoporotic and normal bone lumbar spine. We explored the outcomes of OLIF combined with each of five supplemental fixations: standalone OLIF; OLIF with lateral plate fixation (OLIF+LPF); OLIF with translaminar facet joint fixation and unilateral pedicle and screw fixation (OLIF+TFJF+UPSF); OLIF with unilateral pedicle and screw fixation (OLIF+UPSF); and OLIF with bilateral pedicle and screw fixation (OLIF+BPSF). Under the various working conditions, we calculated the ranges of motion (ROMs) of the normal bone and osteoporosis models, the maximum Mises stresses of the fixation instruments (MMSFIs), and the average Mises stresses on cancellous bone (AMSCBs) .

Results: Compared with the normal bone OLIF model, no demonstrable change in any segmental ROM was apparent. The MMSFIs increased in all five osteoporotic OLIF models. In the OLIF+TFJF+UPSF model, the MMSFIs increased sharply in forward flexion and extension. The stress changes of the OLIF+UPSF, OLIF+BPSF, and OLIF+TFJF+UPSF models were similar; all stresses trended upward. The AMSCBs decreased in all five osteoporotic OLIF models during flexion, extension, lateral bending, and axial rotation. The average stress change of cancellous bone was most obvious under extension. The AMSCBs of the five OLIF models decreased by $14 \%, 23.44 \%, 21.97 \%, 40.56 \%$, and $22.44 \%$ respectively.

Conclusions: For some supplemental fixations, the AMSCBs were all reduced and the MMSFIs were all increased in the osteoporotic model, compared with the OLIF model of normal bone. Therefore, the biomechanical performance of an osteoporotic model may be inferior to the biomechanical performance of a normal model for the same fixation method; in some instances, it may increase the risks of fracture and internal fixation failure.

\section{Background}

Oblique lumbar interbody fusion (OLIF) is an important treatment for disc degeneration and has become very popular among both physicians and patients. OLIF was first proposed by Mayer in 1997 [1]. Later, Silvestre et al. [2] improved the procedure by formally delivering OLIF through a channel between the peritoneum and the psoas major muscle, thus causing minimal invasion of the intervertebral disc space. Compared with other lumbar interbody fusions, the advantages of OLIF include less blood loss and shorter surgery; patients recover quickly and can be rapidly discharged $[3,4]$. During OLIF, the lumbar spine is accessed through a window between the anterior blood vessel and the psoas muscle. Thus, OLIF 
does not damage the posterior structures and can effectively treat intervertebral disc degeneration by retaining more bone mass; this is important in patients with osteoporosis.

Osteoporotic bone is defined as "a type of bone characterized by low bone mass and microstructural degradation caused by bone tissue diseases, the results of which are bone fragility and increased risk of fractures" $[5,6]$. In clinical practice, osteoporotic patients with degenerative lumbar spine disease often require OLIF to increase lumbar stability and reduce the risks of lumbar fracture and failure; the optimal supplemental fixation required when performing OLIF in an osteoporotic patient remains unclear. An appropriate supplemental fixation choice is particularly important in such patients. Screw loosening and extraction have been reported during follow-up of osteoporotic patients who had undergone fusion surgery for internal fixation. Fractures have also been reported; these may require surgical intervention that increases the financial strain for osteoporotic patients [7]. Few biomechanical studies have appeared concerning the effects of osteoporosis on various supplemental fixations co-applied with OLIF. These focused on intraoperative observation, postoperative follow-up, and summaries of prior works $[2,3,8,9]$. Biomechanics clearly has important effects on the physiology, pathology, and surgical repair outcomes of the lumbar spine [10-12]. It is appropriate for clinicians to study the combinations of OLIF with various supplemental fixations for patients with osteoporosis.

The finite element (FE) method is widely used to explore mechanical issues, such as the biomechanics of the lumbar spine [13-17]. Traditional mechanical testing requires clinical specimens or cadavers; however, sourcing of in vitro specimens is difficult and time-consuming. Furthermore, soft tissues such as ligaments and muscles tend to fatigue after death; thus, the results may not consider the important interactions between bone and other tissues. However, FEs can be simulated in the absence of clinical specimens or cadavers, thus partially compensating for the shortage of in vitro data [18-20]. The FE method can be used to simulate the various supplemental fixations, aiding physicians in the development of appropriate treatment plans and selection of optimal surgeries [21]. Thus, considering the advantages of the FE method in the context of a biomechanical problem, we used this method to explore the effects of osteoporosis on the biomechanics of various supplemental fixations co-applied with OLIF.

We built a complete three-dimensional FE model of the L3-S1 lumbar spine and sacrum, then simulated five supplemental fixations that may be co-applied with OLIF. We predicted and compared the ranges of motion (ROMs) of individual segments, the maximum Mises stresses on fixation instruments (MMSFIs), and the average Mises stresses on cancellous bone (AMSCBs). The effects of osteoporosis on the biomechanics of the various supplemental fixations were analyzed.

\section{Methods}

\subsection{Model of the normal lumbar spine}

The FE model used in this study was part (L3-S1) of a previously established and validated spine model, (Figure 1) [16, 17, 22]. In brief, modeling proceeded as follows. A complete geometrical model of the 
lumbar spine was constructed in Mimics 10.0 (Materialise Technologies, Leuven, Belgium) using computed tomography images of a 30-year-old healthy man without any abnormalities. Reconstruction employed Geomagic Studio 10.0 reverse engineering software (Geomagic Inc., NC, USA). HyperMesh 11.0 preprocessing software (Altair Engineering Corp., MI, USA) was used for meshing and assignment of material properties to the vertebral body. Then, FE analysis was performed with the aid of Abaqus 6.11 (Dassault Systems Corp., PA, USA). The complete FE model featured cancellous, cortical, endplate, intervertebral disc, and posterior elements. The cancellous vertebral body was surrounded by a $0.5-\mathrm{mm}$ layer of cortical bone [22,23]. The intervertebral disc featured a nucleus pulposus and an annulus fibrosus in proportions of $44 \%$ and $56 \%$ [24], respectively, reflecting the histological composition. The average space between facets was $0.1 \mathrm{~mm}$. The seven ligaments connecting the vertebrae were modeled as tension-only three-dimensional springs. The detailed material properties of each component of the complete model were derived from the literature and are listed in Table 1.

Table 1

Normal bone: material properties and elements of the lumbar spine model and the implants.

\begin{tabular}{|llll}
\hline Component & Young's modulus $(\mathrm{MPa})$ & Poisson's ratio & Element types \\
\hline Cortical & $12000(7920)$ & 0.3 & C3D8R \\
\hline Cancellous & $100(33)$ & 0.3 & C3D4 \\
\hline Posterior element & $3500(2310)$ & 0.3 & C3D4 \\
\hline Endplate & $24(16)$ & 0.4 & C3D8R \\
\hline Sacrum & 5000 & 0.2 & C3D4 \\
\hline Cage & 3600 & 0.25 & C3D8R \\
\hline Screws and rods & 110000 & 0.3 & C3D8R \\
\hline Lateral plate & 110000 & 0.3 & C3D8R \\
\hline Facet cartilage & Neo-Hookean, C10 = 2 & C3D8RH \\
\hline Annulus ground & Mooney-Rivlin, C1 = 0.18, C2 = 0.045 & C3D8RH \\
\hline Nucleus pulposus & Mooney-Rivlin, C1 = 0.12, C2 = 0.03 & C3D8RH \\
\hline Annulus fibers & Calibrated stress-strain curves & Spring \\
\hline Seven ligaments & Calibrated deflection-force curves & Spring \\
\hline $\begin{array}{l}\text { Seven ligaments: anterior longitudinal ligament; posterior longitudinal ligament; intertransverse } \\
\text { ligament; ligamentum flavum; supraspinous ligament; interspinous ligament; capsular ligament. }\end{array}$
\end{tabular}

\subsection{OLIF surgical models}

We evaluated five supplemental fixations for osteoporotic lumbar spines: standalone OLIF; OLIF with lateral plate fixation (OLIF+LPF); OLIF with translaminar facet joint fixation and unilateral pedicle and 
screw fixation (OLIF+TFJF+UPSF); OLIF with unilateral pedicle and screw fixation (OLIF+UPSF); and OLIF with bilateral pedicle and screw fixation (OLIF+BPSF). We simulated surgery on the L4-L5 segment (Figure 2) because this is the segment most often affected in asymptomatic individuals [14, 25, 26]. During the simulations, the entire nucleus pulposus and part of the annulus fibrosus were removed (Figure 1). We then created pedicle screws (diameter, $6.5 \mathrm{~mm}$; length, $45 \mathrm{~mm}$ ), a rod (diameter, $6.0 \mathrm{~mm}$ ), and a cage (length, $45 \mathrm{~mm}$; width, $22 \mathrm{~mm}$; average width, $9.5 \mathrm{~mm}$; surface area, $28.21 \mathrm{~cm}^{2}$ ) in SolidWorks. We introduced the cage to the intervertebral discs of the L4-L5 segment, imported IGES files of the screws and vertebrae into HyperMesh, generated holes via Boolean operations, and added different fixation devices to the L4-L5 segments (Figure 1). Notably, because the rigid fixation system was simulated, the screw-rod and screw-side plate interacted through a common node, while the screwvertebrae and cage-endplate interacted through a "Tie" constraint.

\subsection{Osteoporotic vertebral material properties}

According to a previous study [27], osteoporosis changes the elastic moduli of all bones in the vertebral body without significant changes in other material parameters. The thickness of lumbar spine cortical bone does not significantly change. Thus, when creating the osteoporotic FE model, the modulus of elasticity was reduced by $33 \%$ for cortical bone and the endplate and posterior structures; it was reduced by $66 \%$ for all cancellous bone structures [27-29]. All other material parameters were unchanged. The vertebral and posterior structures were considered to be isotropic and homogeneously elastic. The material properties and elements of the osteoporotic components are listed in Table 2.

Table 2 Osteoporotic bone: material properties and elements of the lumbar spine model.

\begin{tabular}{|llll|}
\hline Component & Young's modulus (MPa) & Poisson's ratio & Element types \\
\hline Cortical & $7920(\downarrow 33 \%)$ & 0.3 & C3D8R \\
\hline Cancellous & $33(\downarrow 66 \%)$ & 0.3 & C3D4 \\
\hline Posterior element & $2310(\downarrow 33 \%)$ & 0.3 & C3D4 \\
\hline Endplate & $16(\downarrow 33 \%)$ & 0.4 & C3D8R \\
\hline
\end{tabular}

\subsection{Boundary and load conditions}

Coupling points were set in the centers of the upper and lower endplate surfaces of each vertebral body; seven such points were set in the L3-S1 segment. Connector elements were created through the coupling points. As shown in Figure 3, the follower load is a physiological compressive load along the axis of the spine. A follower load of $500 \mathrm{~N}$ was applied to each level of the lumbar spine through the abovementioned connector elements. The follower loads were applied as suggested in the literature [17, 30]. Under a follower load of $500 \mathrm{~N}$, a moment load of $7.5 \mathrm{~N} \cdot \mathrm{m}$ was applied to the upper surface of the upper endplate of $L 3$ to simulate six different postures: flexion, extension, left bending, right bending, left 
axial rotation, and right axial rotation. During the entire loading process, the translational and rotational degrees of freedom of $S 1$ were limited in the $X, Y$, and $Z$ axes [31].

\section{Results}

\subsection{ROMs}

Figure 4 shows the ROMs of the L3-S1 segments of different models (five OLIF models each for normal and osteoporotic bone) in the six postures. OLIF for patients with osteoporotic or normal bone significantly reduced the ROM of the L4-L5 surgical segment. The overall segmental ROM of the normal model decreased, compared with the overall segmental ROM of the osteoporotic model, principally because the ROM of the operative segment decreased. When the various supplemental fixations were applied, the osteoporotic OLIF models revealed no obvious change in the ROM of any segment, compared with the ROM of the normal bone OLIF model.

\subsection{MMSFIs under normal and osteoporotic conditions}

The peak Mises stresses on the various supplemental fixation instruments (in different postures) of both models are shown in Figure 5. Notably, MMSFls were detected in both the osteoporotic and normal bone models under various working conditions. The MMSFIs in osteoporotic bone were larger than the MMSFIs in the normal bone surgical model. Under flexion, MMSFIs were detected on the supplemental fixation instruments of both the osteoporotic and normal bone surgery models (Figure 6). The MMSFIs of the OLIF+UPSF and OLIF+BPSF models increased by $16.56 \%$ (from 32.91 to 38.36 ) and $16.47 \%$ (from 18.94 to $22.06 \mathrm{MPa}$ ), respectively. In the OLIF+TFJF+UPSF model, the MMSFI increased sharply from 29.88 to $59.00 \mathrm{MPa}$; in the OLIF+LPF model, the MMSFI changed slightly from 56.89 to $61.22 \mathrm{MPa}$ (a $7.61 \%$ increase). Similarly, the stresses on the supplemental fixation instruments of the osteoporotic model were highest after extension, as in the normal bone model (Figure 5). In the OLIF+TFJF+UPSF model, the MMSFI increased sharply with decreasing bone mass (from 59.11 to $114.40 \mathrm{MPa}$ ). In the OLIF+LPF model, the MMSFI decreased from 101.40 to $116.01 \mathrm{MPa}$ (a reduction of $14.41 \%$ ). However, in the OLIF+UPSF and OLIF+BPSF models, the MMSFIs were similar to the values for flexion. Compared with the normal bone model, the osteoporotic model imposed higher stresses on the supplemental fixation instruments.

The maximum stresses on the supplemental fixation instruments during lateral bending and axial rotation are shown in Figure 5. Both the osteoporotic and normal models exhibited MMSFIs. Comparisons revealed that the stress changes of the OLIF+UPSF, OLIF+BPSF, and OLIF+TFJF+UPSF models during lateral bending were similar to the values on flexion and extension; all stresses trended upward. The changes were most obvious in the OLIF+TFJF+UPSF model. However, in the OLIF+LPF model (compared 
with the normal bone surgery model), the stresses on the supplemental fixation instruments increased by $3.12 \%$ (from 94.78 to $97.74 \mathrm{MPa}$ ) and $1.37 \%$ (from 114.62 to $116.19 \mathrm{MPa}$ ), respectively.

\subsection{AMSCBs}

As shown in Figure 6, under different loads, the AMSCBs of the five OLIF osteoporotic models decreased, compared with the values of the normal bone model. AMSCBs were evaluated because it is important to eliminate stress concentrations caused by perforations of cancellous bone. In the osteoporotic model, the AMSCBs were markedly altered (Figure 6). Under flexion, the comparison indicated that the AMSCB decreased from 0.133 to $0.121 \mathrm{MPa}$ in the standalone OLIF model; it decreased from 0.121 to $0.101 \mathrm{MPa}$ in the OLIF+UPSF model, 0.109 to $0.093 \mathrm{MPa}$ in the OLIF+BPSF model, 0.14 to $0.111 \mathrm{MPa}$ in the OLIF+LPF model, and 0.119 to $0.102 \mathrm{MPa}$ in the OLIF+TFJF+UPSF model. Notably, the average stress changes on cancellous bone were most obvious under extension. The AMSCBs of the five OLIF models decreased by $14 \%, 23.44 \%, 21.97 \%, 40.56 \%$, and $22.44 \%$, respectively. Of these, the OLIF+LPF change was most obvious: from 0.18 to $0.107 \mathrm{MPa}$. Importantly, during lateral bending and axial rotation, the AMSCBs were similar to the values under flexion and extension. Compared with the values of the normal bone model, the AMSCBs decreased to varying extents.

\section{Discussion}

OLIF is favored by spinal surgeons because it is associated with less bleeding, a shorter operative time, and faster recovery, compared with other lumbar disc fusion surgeries [32,33]. Although OLIF can achieve excellent results and is widely used in practice, it can destabilize the spine and increase the risks of cage subsidence and fracture $[8,34]$. In patients with osteoporosis, these risks may be greater because of the reduced bone mass and increased degradation of bone, compared with those characteristics in patients with normal bone mass; osteoporotic bone is more brittle. In earlier reports, patients with osteoporosis were prone to complications such as screw loosening and extraction, which increased internal fixation failure [35-37]. However, the effects of OLIF co-applied with various supplemental fixations in osteoporotic patients remain unknown. Therefore, we built FE models of osteoporotic and normal spines, then applied various supplemental fixations to determine their biomechanical responses.

To explore the effects of osteoporosis on various supplemental fixations that may be co-applied with OLIF, we established and verified a three-dimensional, nonlinear complete FE (L3-S1) model. Then, we endowed each part of the lumbar spine with osteoporotic features to establish a three-dimensional, nonlinear, osteoporotic FE model. Appropriate modifications were made at the L4-L5 level; we simulated five OLIF models using different fixed instruments.

After a patient has undergone OLIF surgery, the stability of the surgical segment is an important index of rehabilitation that greatly concerns clinicians [38] because instability is usually associated with various complications such as intervertebral interstitial inflammation, cage subsidence, reduced intervertebral disc height, and vertebral body non-fusion. Lu et al. [4] used an FE model to explore the biomechanical properties of four LIF surgeries (PLIF, TLIF, XLIF, and OLIF). The ROMs of the surgical segments were 
reduced. Chen et al. [39] established single-segment lateral interbody fusion surgical models; they found that, compared with the complete model, the ROMs decreased by $76.84-97.97 \%$. Oxland et al. [40] reviewed the biomechanical characteristics of LIF surgeries. The maximum ROM reduction at the index level was $90 \%$. We found that OLIF co-applied with various supplemental fixations in osteoporotic patients significantly reduced the ROM of the L4-L5 surgical segment; thus, it afforded good surgical site stability. As shown in Figure 5, the ROMs of the five supplemental fixation instruments in different positions did not differ markedly from the ROMs of normal bone, either for the surgical segment (L4-L5) or the non-fused segment. These results indicated that osteoporosis did not greatly affect the ROM of the lumbar spine, consistent with the findings in previous studies [41]. Therefore, OLIF surgery using the same fixation modality in osteoporotic patients does not affect the vertebral body ROM to a greater extent than in patients with normal bone quality. Previous studies found that a reduced spinal ROM was usually associated with disc degeneration [42, 43]. Because the intervertebral discs of the osteoporotic spine are not markedly degenerative, it is unsurprising to find that their mobility is not significantly affected.

In the osteoporotic model, the stresses on the vertebrae and supplemental fixations changed greatly, compared with stresses in the normal model). Under axial compression, the displacement of osteoporotic vertebrae is greater than the displacement of normal vertebrae; however, considering the lower strength of osteoporotic vertebrae, as well as the small displacements of the screw and rod, the load is transferred to the screw and rod. Under flexion, osteoporotic vertebrae are less stiff than normal vertebrae and exhibit deformation; screw displacement is reduced and more torque is generated between the vertebrae and the screw, imparting high-level stress to the screw. Moreover, the increased vertebral displacement enhances screw and rod displacement; the rods bend more and thus experience higher stresses. Similarly, under extension, the osteoporotic vertebral body is softer and more deformed than the normal vertebral body; a small screw displacement may increase the distance between the vertebral body and the screw, concentrating stress on the screw. Considering the softer osteoporotic vertebral body, compared with normal bone, greater stresses are imparted to the supplemental fixation instruments; this is consistent with the above findings for the rod.

Notably, during standalone OLIF, only the average stress on the vertebrae was analyzed; no supplemental fixation instrument was placed. Compared with normal lumbar spine surgery, standalone OLIF produced less average stress, similar to the results in other fixation systems. This is presumably because osteoporotic vertebrae are softer than normal vertebrae; thus, they impart less stress. The stress levels on the vertebrae and the supplemental fixations in the osteoporotic model are reduced and increased, respectively, in the various postures, compared with those values in the normal bone model; these findings are consistent with the results of previous studies [44]. Therefore, the biomechanical properties of the osteoporotic model are less robust than the properties of the normal model when the same supplemental fixation method is employed.

We found that osteoporosis affected OLIF to various extents, depending on the chosen supplemental fixation system. Fixation affords strong support and can prevent fractures. In the osteoporotic model, stresses on the proximal-junction vertebrae are reduced. However, the stress on a proximal fixation 
system increases; stress becomes concentrated on the contact interface between the cone and the screw, which is associated with an increased risk of internal fixation failure. Osteoporosis reduces the vertebral elastic modulus and tensile strength. Such changes may increase the relative displacement between the vertebrae and the fixation system in the same radial direction; this may cause the internal fixation device to loosen or rupture. A high pressure at the contact interface between the vertebrae and the screw can trigger bone destruction, such as a fracture. The fixation system chosen and the vertebral strength should be considered when performing OLIF in osteoporotic patients. Firm fixation and a strong vertebral body are essential for the long-term maintenance of fractures repaired after exposure to high stress; they are also essential for reducing the risk of internal fixation failure.

The structure of the lumbar spine is complex; any FE model will feature some limitations and undesirable simplifications. First, our FE model of the lumbar spine was based on geometrical information from one person. The osteoporotic FE model was constructed by ignoring individual differences and reducing the elastic moduli of the endplate, as well as the cortical, cancellous, and posterior elements, by specific proportions. Second, although FE analysis affords many advantages for assessment of biomechanics, compared with in vitro experiments, the inability to reconstruct muscles is a common problem experienced by all current lumbar FE models. Furthermore, FE methods do not closely simulate the true geometry of ligaments; these are simplified to one-dimensional non-linear springs. Finally, the results of FE analysis reflect only the post-surgical condition, rather than the long-term postoperative status. Despite these limitations, the response parameters of our lumbar spine FE model are consistent with published in vitro experimental data concerning spinal biomechanics. Therefore, clinicians may find our results useful.

\section{Conclusions}

We used three-dimensional, nonlinear FE analysis to simulate OLIF surgery in patients with osteoporosis and degenerative disease; we evaluated the utilities of various supplemental fixation methods. The stress distribution patterns of cancellous bone and the fixation instruments were similar when osteoporotic and normal bone models were compared under different bone conditions. Of the six postural conditions evaluated, the MMSFIs and AMSCBs decreased and increased, respectively, in the osteoporotic bone mass model, compared with the normal bone mass model. From a biomechanical perspective, osteoporosis impacts the choice of supplementary fixation during OLIF. Appropriate treatment of osteoporosis is essential to reduce the risk of internal fixation failure.

\section{Abbreviations}

OLIF

Oblique lumbar interbody fusion

FE

finite element

OLIF+LPF

OLIF with lateral plate fixation

Page 10/20 
OLIF+TFJF+UPSF

OLIF with translaminar facet joint fixation and unilateral pedicle and screw fixation

OLIF+UPSF

OLIF with unilateral pedicle and screw fixation

OLIF+BPSF

OLIF with bilateral pedicle and screw fixation

Rom

ranges of motion

MMSFIs

the maximum Mises stresses of the fixation instruments

AMSCBs

the average Mises stresses on cancellous bone

\section{Declarations}

\section{Ethics approval and consent to participate}

Our study was approved by the institutional ethics committee of the Tianjin Hospital. All procedures were performed in accordance with relevant guidelines. Written informed consent was obtained from all participants included in this study.

\section{Consent for publication}

Not Applicable.

\section{Availability of data and materials}

The datasets used and/or analyzed during the current study are available from the corresponding author on reasonable request.

\section{Competing interests}

None of the authors had any potential conflicts of interest to declare.

\section{Funding}

This study was supported by The National Key Research and Development Program of China (2020YFC1107400) and National Orthopaedic and Sports Rehabilitation Clinical Medicine Research Center Innovation Fund. The authors have no personal financial or institutional interest in any of the drugs, materials, or devices described in this article.

\section{Authors' contributions}


ZL, ZG, XC, YR, and QY carried out the model development and simulation, data analysis and drafted the manuscript. ZL, QY and CD participated in the study design. CC, ZG, ZL, XS and XM participated in revising the manuscript. All authors read and approved the final manuscript.

\section{Acknowledgements}

Not applicable.

The English in this document has been checked by at least two professional editors, both native speakers of English. For a certificate, please see:

http://www.textcheck.com/certificate/eezYq4

\section{References}

1. Mayer HM. A new microsurgical technique for minimally invasive anterior lumbar interbody fusion. Spine. 1997;22(6):691-9. discussion 700.

2. Silvestre C, Mac-Thiong JM, Hilmi R, Roussouly P. Complications and Morbidities of Mini-open Anterior Retroperitoneal Lumbar Interbody Fusion: Oblique Lumbar Interbody Fusion in 179 Patients. Asian spine journal. 2012;6(2):89-97.

3. Mobbs RJ, Phan K, Malham G, Seex K, Rao PJ. Lumbar interbody fusion: techniques, indications and comparison of interbody fusion options including PLIF, TLIF, MI-TLIF, OLIF/ATP, LLIF and ALIF. Journal of spine surgery (Hong Kong). 2015;1(1):2-18.

4. Lu T, Lu Y. Comparison of Biomechanical Performance Among Posterolateral Fusion and Transforaminal, Extreme, and Oblique Lumbar Interbody Fusion: A Finite Element Analysis. World neurosurgery. 2019;129:e890-9.

5. Osteoporosis prevention, diagnosis, and therapy. Jama. 2001;285(6):785-95.

6. Office of the Surgeon G: Reports of the Surgeon General. In: Bone Health and Osteoporosis: A Report of the Surgeon General. edn. Rockville (MD): Office of the Surgeon General (US); 2004.

7. Jimbo S, Kobayashi T, Aono K, Atsuta Y, Matsuno T. Epidemiology of degenerative lumbar scoliosis: a community-based cohort study. Spine. 2012;37(20):1763-70.

8. Quillo-Olvera J, Lin GX, Jo HJ, Kim JS. Complications on minimally invasive oblique lumbar interbody fusion at L2-L5 levels: a review of the literature and surgical strategies. Annals of translational medicine. 2018;6(6):101.

9. Li JX, Phan K, Mobbs R. Oblique Lumbar Interbody Fusion: Technical Aspects, Operative Outcomes, and Complications. World neurosurgery. 2017;98:113-23.

10. Aulisa L, Pitta L, Padua R, Ceccarelli E, Aulisa A, Leone A. Biomechanics of the spine. Rays. 2000;25(1):11-8.

11. Wilder DG, Pope MH, Frymoyer JW. The biomechanics of lumbar disc herniation and the effect of overload and instability. J Spinal Disord. 1988;1(1):16-32. 
12. Stemper BD, Baisden JL, Yoganandan N, Shender BS, Maiman DJ. Mechanical yield of the lumbar annulus: a possible contributor to instability: Laboratory investigation. Journal of neurosurgery Spine. 2014;21(4):608-13.

13. Liu X, Ma J, Park P, Huang X, Xie N, Ye X. Biomechanical comparison of multilevel lateral interbody fusion with and without supplementary instrumentation: a three-dimensional finite element study. BMC Musculoskelet Disord. 2017;18(1):63.

14. Ruberté LM, Natarajan RN, Andersson GB. Influence of single-level lumbar degenerative disc disease on the behavior of the adjacent segments--a finite element model study. Journal of biomechanics. 2009;42(3):341-8.

15. Ambati DV. Wright EK, Jr., Lehman RA, Jr., Kang DG, Wagner SC, Dmitriev AE: Bilateral pedicle screw fixation provides superior biomechanical stability in transforaminal lumbar interbody fusion: a finite element study. The spine journal: official journal of the North American Spine Society 2015, 15(8):1812-22.

16. Du CF, Yang N, Guo JC, Huang YP, Zhang C. Biomechanical response of lumbar facet joints under follower preload: a finite element study. BMC Musculoskelet Disord. 2016;17:126.

17. Du C, Mo Z, Tian S, Wang L, Fan J, Liu S et al: Biomechanical investigation of thoracolumbar spine in different postures during ejection using a combined finite element and multi-body approach. International journal for numerical methods in biomedical engineering 2014, 30(11):1121-1131.

18. Godzik J, Kalb S, Reis MT, Reyes PM, Singh V, Newcomb A et al: Biomechanical evaluation of interbody fixation with secondary augmentation: lateral lumbar interbody fusion versus posterior lumbar interbody fusion. Journal of spine surgery (Hong Kong) 2018, 4(2):180-186.

19. Reis MT, Reyes PM, Bse, Altun I, Newcomb AG, Singh V et al: Biomechanical evaluation of lateral lumbar interbody fusion with secondary augmentation. Journal of neurosurgery Spine 2016, 25(6):720-726.

20. Godzik J, Martinez-Del-Campo E, Newcomb A, Reis MT, Perez-Orribo L, Whiting AC et al: Biomechanical Stability Afforded by Unilateral Versus Bilateral Pedicle Screw Fixation with and without Interbody Support Using Lateral Lumbar Interbody Fusion. World neurosurgery 2018, 113:e439-e445.

21. Muheremu A, Li H, Ma J, Ma Y, Ma Y. Establishment of a three-dimensional finite element model of severe kyphotic deformity secondary to ankylosing spondylitis. J Int Med Res. 2017;45(2):639-46.

22. Du CF, Cai XY, Gui W, Sun MS, Liu ZX, Liu CJ et al: Does oblique lumbar interbody fusion promote adjacent degeneration in degenerative disc disease: A finite element analysis. Computers in biology and medicine 2021, 128:104122.

23. Hadley C, Awan OA, Zoarski GH. Biomechanics of vertebral bone augmentation. Neuroimaging Clin $\mathrm{N}$ Am. 2010;20(2):159-67.

24. Schmidt H, Heuer F, Drumm J, Klezl Z, Claes L, Wilke HJ. Application of a calibration method provides more realistic results for a finite element model of a lumbar spinal segment. Clinical biomechanics (Bristol. Avon) 2007;22(4):377-84. 
25. Elfering A, Semmer N, Birkhofer D, Zanetti M, Hodler J, Boos N. Risk factors for lumbar disc degeneration: a 5-year prospective MRI study in asymptomatic individuals. Spine. 2002;27(2):12534.

26. Boden SD, Davis DO, Dina TS, Patronas NJ, Wiesel SW: Abnormal magnetic-resonance scans of the lumbar spine in asymptomatic subjects. A prospective investigation. The Journal of bone and joint surgery American volume 1990, 72(3):403-408.

27. Polikeit A, Nolte LP, Ferguson SJ. The effect of cement augmentation on the load transfer in an osteoporotic functional spinal unit: finite-element analysis. Spine. 2003;28(10):991-6.

28. Salvatore G, Berton A, Giambini H, Ciuffreda M, Florio P, Longo UG et al: Biomechanical effects of metastasis in the osteoporotic lumbar spine: A Finite Element Analysis. BMC musculoskeletal disorders 2018, 19(1):38.

29. Su X, Shen H, Shi W, Yang H, Lv F, Lin J. Dynamic characteristics of osteoporotic lumbar spine under vertical vibration after cement augmentation. American journal of translational research. 2017;9(9):4036-45.

30. Ayturk UM, Garcia JJ, Puttlitz CM. The micromechanical role of the annulus fibrosus components under physiological loading of the lumbar spine. J Biomech Eng. 2010;132(6):061007.

31. Shirazi-Adl SA, Shrivastava SC, Ahmed AM. Stress analysis of the lumbar disc-body unit in compression. A three-dimensional nonlinear finite element study. Spine. 1984;9(2):120-34.

32. Lee YS, Park SW, Kim YB. Direct lateral lumbar interbody fusion: clinical and radiological outcomes. J Korean Neurosurg Soc. 2014;55(5):248-54.

33. Alimi M, Hofstetter CP, Tsiouris AJ, Elowitz E, Härtl R. Extreme lateral interbody fusion for unilateral symptomatic vertical foraminal stenosis. European spine journal. 2015;24(Suppl 3):346-52. o t and the European Section of the Cervical Spine Research Society.

34. Abe K, Orita S, Mannoji C, Motegi H, Aramomi M, Ishikawa T et al: Perioperative Complications in 155 Patients Who Underwent Oblique Lateral Interbody Fusion Surgery: Perspectives and Indications From a Retrospective, Multicenter Survey. Spine 2017, 42(1):55-62.

35. Kim JB, Park SW, Lee YS, Nam TK, Park YS, Kim YB. The Effects of Spinopelvic Parameters and Paraspinal Muscle Degeneration on S1 Screw Loosening. J Korean Neurosurg Soc. 2015;58(4):35762.

36. Wu ZX, Gong FT, Liu L, Ma ZS, Zhang Y, Zhao X et al: A comparative study on screw loosening in osteoporotic lumbar spine fusion between expandable and conventional pedicle screws. Archives of orthopaedic and trauma surgery 2012, 132(4):471-476.

37. Halvorson TL, Kelley LA, Thomas KA. Whitecloud TS, 3rd, Cook SD: Effects of bone mineral density on pedicle screw fixation. Spine 1994, 19(21):2415-20.

38. Zhang Z, Fogel GR, Liao Z, Sun Y, Liu W. Biomechanical Analysis of Lateral Lumbar Interbody Fusion Constructs with Various Fixation Options: Based on a Validated Finite Element Model. World neurosurgery. 2018;114:e1120-9. 
39. Chen YL, Lai OJ, Wang Y, Ma WH, Chen QX. The biomechanical study of a modified lumbar interbody fusion-crenel lateral interbody fusion (CLIF): a three-dimensional finite-element analysis. Comput Methods Biomech BioMed Eng. 2020;23(9):548-55.

40. Oxland TR, Lund T. Biomechanics of stand-alone cages and cages in combination with posterior fixation: a literature review. European spine journal. 2000;9(Suppl 1(Suppl 1):95-101. o $t$ and the European Section of the Cervical Spine Research Society.

41. Yang Z, Griffith JF, Leung PC, Lee R. Effect of osteoporosis on morphology and mobility of the lumbar spine. Spine. 2009;34(3):E115-21.

42. Mimura M, Panjabi MM, Oxland TR, Crisco JJ, Yamamoto I, Vasavada A. Disc degeneration affects the multidirectional flexibility of the lumbar spine. Spine. 1994;19(12):1371-80.

43. Fujiwara A, Lim TH, An HS, Tanaka N, Jeon CH, Andersson GB et al: The effect of disc degeneration and facet joint osteoarthritis on the segmental flexibility of the lumbar spine. Spine 2000, 25(23):3036-3044.

44. Wang T, Zhao Y, Cai Z, Wang W, Xia Y, Zheng G et al: Effect of osteoporosis on internal fixation after spinal osteotomy: A finite element analysis. Clinical biomechanics (Bristol, Avon) 2019, 69:178-183.

\section{Figures}

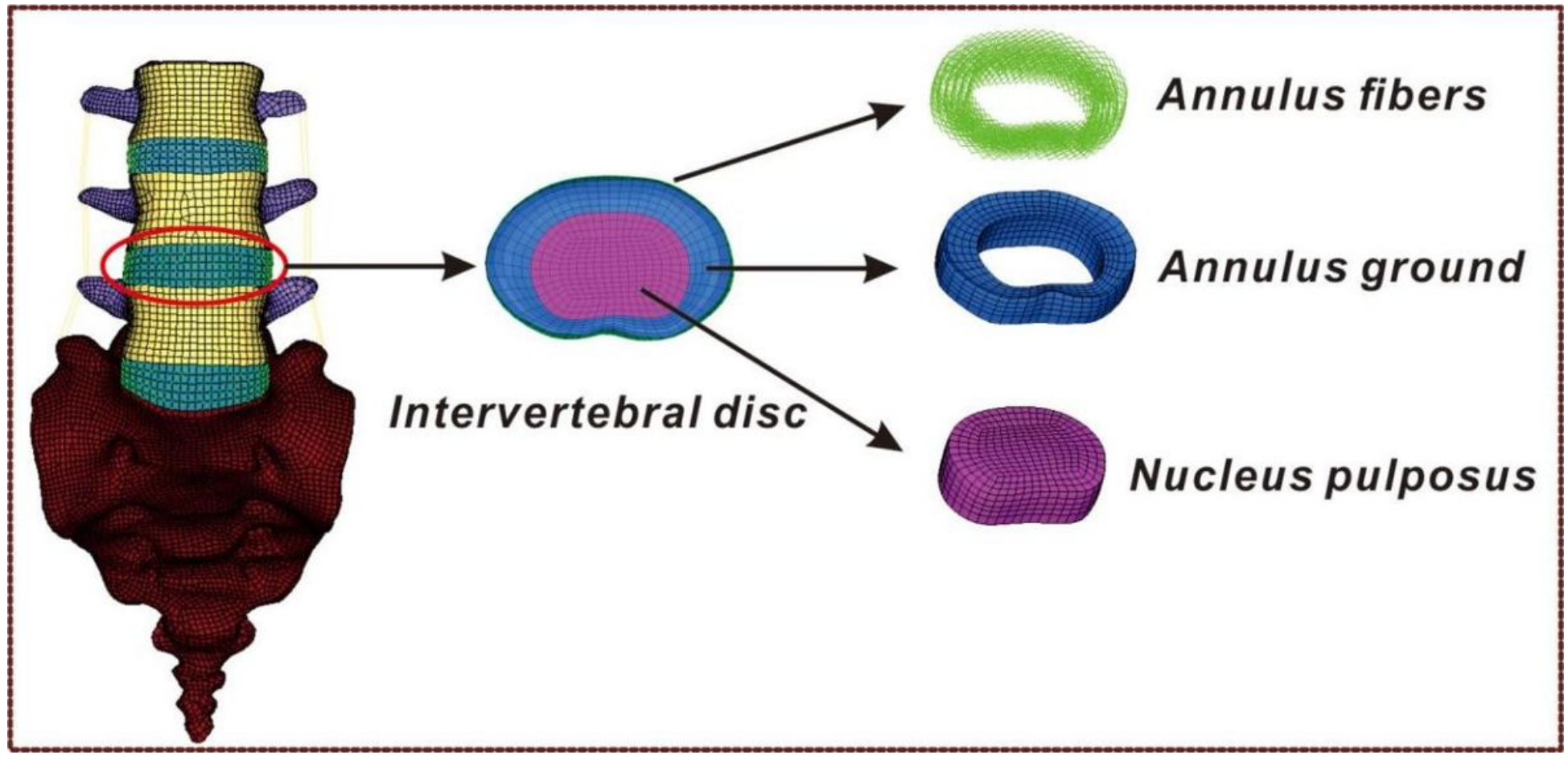

Figure 1

Three-dimensional, nonlinear finite element model of the lumbar spine (L3-S1). 


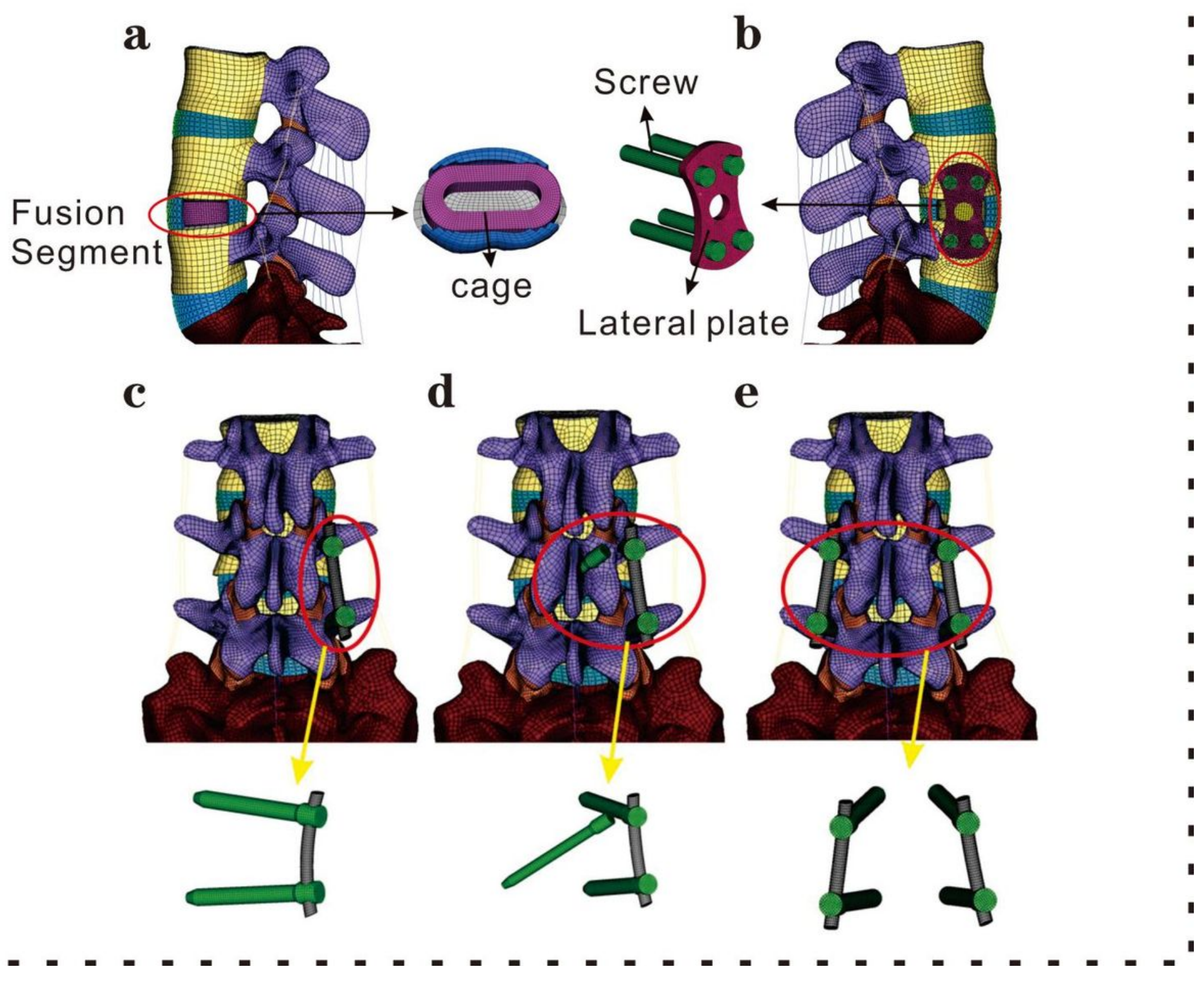

Figure 2

Five OLIF lumbar spine FE models.

a: Standalone OLIF;

b: OLIF+LPF; OLIF with lateral plate fixation;

c: OLIF+UPSF; OLIF with unilateral pedicle and rod fixation;

d: OLIF+TFJF+UPSF; OLIF with translaminar facet joint fixation and unilateral pedicle and rod fixation;

e: OLIF+BPSF; OLIF with bilateral pedicle and screw and rod fixation. 


\section{Validation}

Flexion moments of $8 \mathrm{~N} \cdot \mathrm{m}$

Extension moments of $6 \mathrm{~N} \cdot \mathrm{m}$ Lateral-bending moments of $\pm 6 \mathrm{~N} \cdot \mathrm{m}$

Torsional moments of $\pm 4 \mathrm{~N} \cdot \mathrm{m}$

Follower load $500 \mathrm{~N}, 1200 \mathrm{~N}$

\section{Osteoporosis model}

$7.5 \mathrm{~N} \cdot \mathrm{m}$ moment

Follower load $500 \mathrm{~N}$

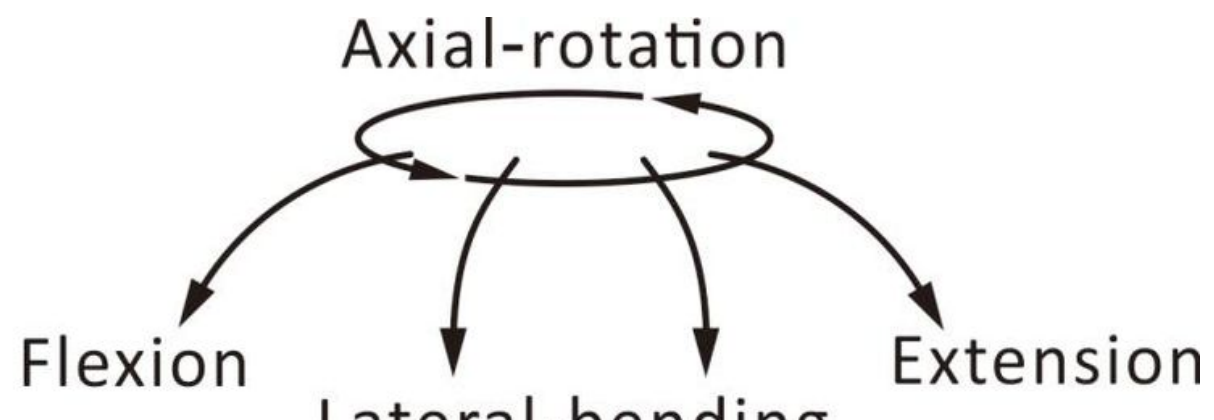

Fixed

constraint

\section{Fusion model}

Hybrid loading conditions

Follower load $500 \mathrm{~N}$

Figure 3

Schematic of torque and follower loads applied to the lumbar spine (L3-S1). 


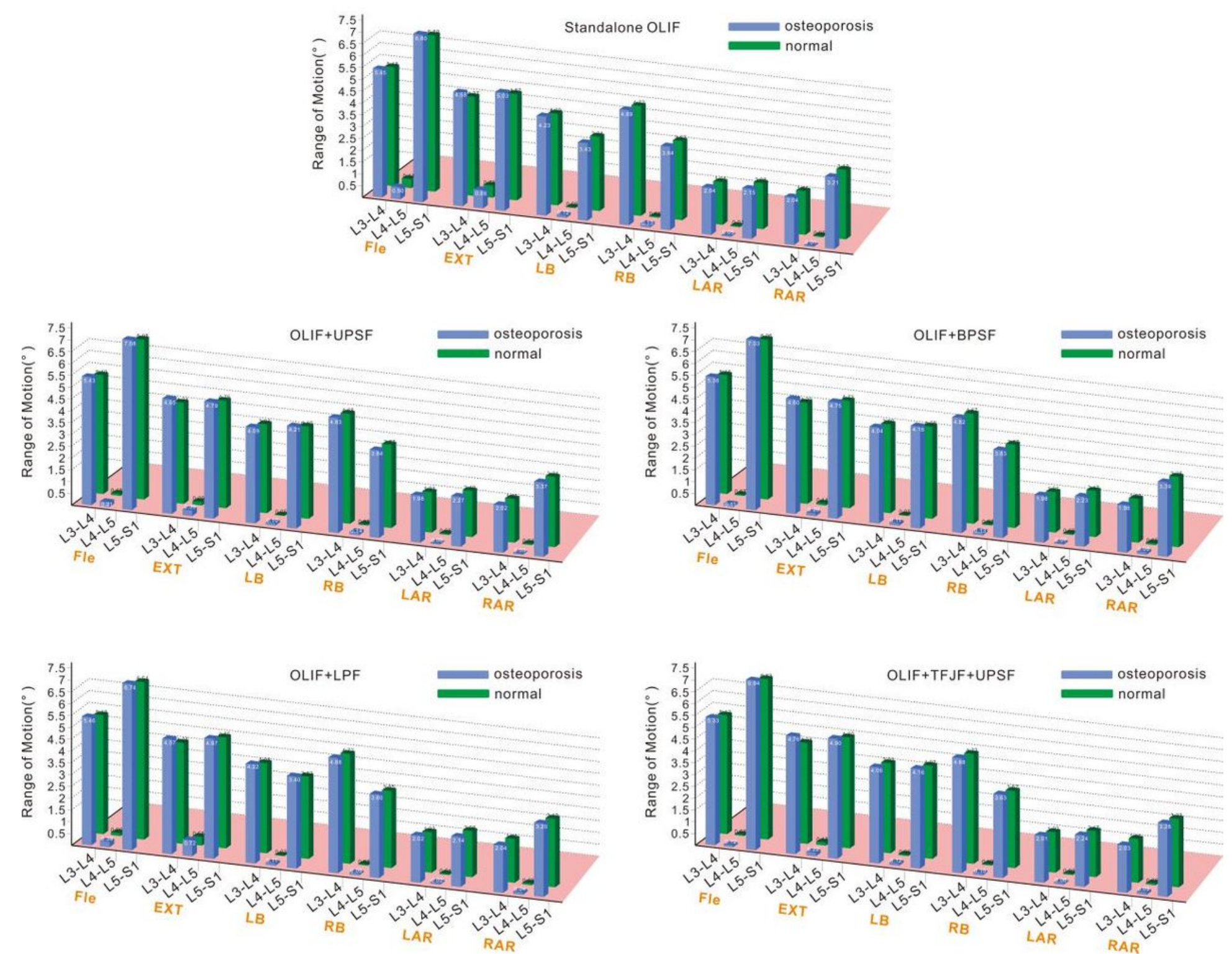

Figure 4

ROMs of various segments of osteoporotic and normal bones. 


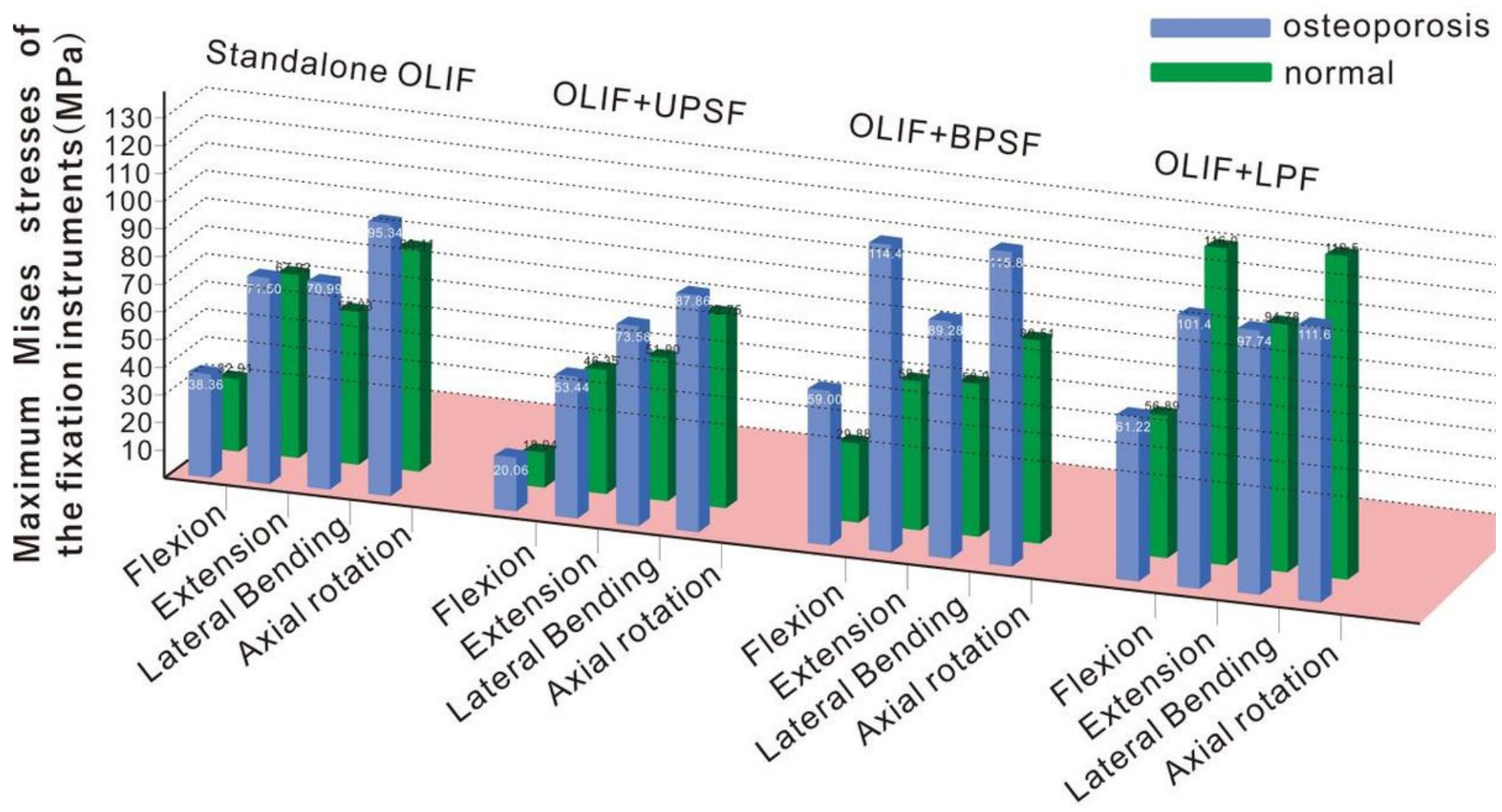

Figure 5

Maximum Mises stresses of the fixation instruments (MMSFIs) of normal and osteoporotic bones.

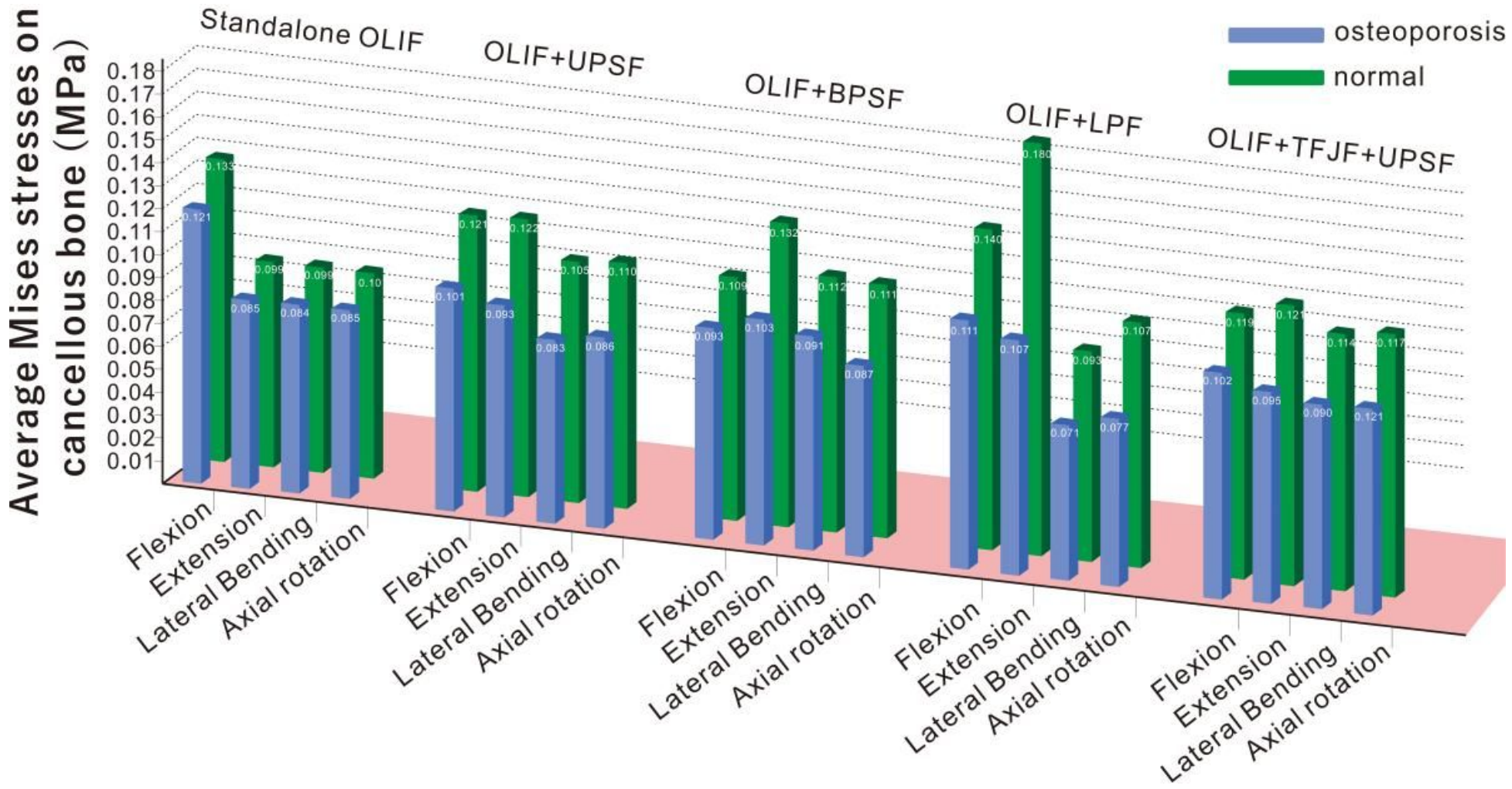

Figure 6 
Average Mises stresses (AMSCBs) of cancellous normal and osteoporotic bones. 